\title{
КОНЦЕПТУАЛЬНЫЕ ОСНОВЫ УПРАВЛЕНИЯ РИСКАМИ ТОРГОВЫХ ПРЕДПРИНИМАТЕЛЬСКИХ СТРУКТУР В УСЛОВИЯХ НЕОПРЕДЕЛЕННОСТИ И ВОЗДЕЙСТВИЯ ПОВЫШЕННЫХ ВНЕШНИХ РИСКОВ
}

\author{
(c) 2021 Кунин Владимир Александрович \\ доктор экономических наук, доцент \\ (c) 2021 Михайловский Дмитрий Александрович \\ аспирант \\ коммерческий директор, ЗАО «Альянс-ГРУПП»
}

Санкт-Петербургский университет технологий управления и экономики, Россия, Санкт-Петербург

В настоящей статье предлагается концепция сквозного управления рисками торговых предпринимательских структур, включающая в себя базисные принципы и методику сквозного управления рисками на всех стадиях их активации и воздействия, а также кейс-метод формирования информационной базы данных. Применение этого метода обеспечивает структуризацию задач, методов и мер по управлению рисками в соответствии с видом, стадией и владельцем риска. В работе также предложен и аргументирован перечень цифровых сервисов, повышающих эффективность процессов риск-менеджмента, обоснована целесообразность применения гибкой методологии Agile, обеспечивающей удобный и легкий мониторинг процесса решения задач управления рисками в условиях неопределённости, и раскрывается алгоритм применения этой методологии.

Ключевые слова: риск, неопределённость, торговая предпринимательская структура, цифровые технологии, кейс, сквозное управление.

\section{Введение}

В современной научной литературе вопросам риск-менеджмента посвящено множество работ, в рамках которых рассматриваются различные аспекты, связанные с совершенствованием процессов управления рисками.

Однако стремительные темпы развития современных технологий заставляют исследователей заново пересматривать ранее разработанные методы управления рисками, внедряя в риск-менеджмент современные цифровые инструменты. В соответствии с современными подходами к организации риск - менеджмента система управления рисками должна быть органично интегрирована в общую систему менеджмента предприятия и не функционировать как обособленный инструмент управления [1-5].

При этом, цифровые технологии дают возможность совершенствовать методы и алгоритмы управления рисками за счет применения новых цифровых инструментов в значительной степени повышающих эффективность и возможности риск-менеджмента. Более того, развитие облачных технологий сделало целесообразным и доступным внедрение современных методов управления рисками в предпринимательских структурах, имеющих ограниченные инвестиционные ресурсы для разработки и внедрения собственных сервисов и программ, что существенно расширяет возможности цифровой трансформации процессов управления рисками. Такая трансформация особенно важна в условиях неопределённости и повышенного воздействия внешних рисков, когда номенклатура реализующихся рисков расширяется и увеличивается объём информации, необходимой для идентификации рисков и разработки мер по их снижению [6-8].

В этой связи в настоящей работе предлагается концепция сквозного управления частными рисками торговых предпринимательских структур включающая базисные принципы и методику сквозного управления рисками, а также кейсовый метод формирования информационной базы данных, используемых для обеспечения удобного и эффективного риск - менеджмента.

Методика сквозного управления частными рисками торговых предпринимательских структур

Важным элементом классификации предпринимательских рисков является их разделение на частные риски и стратегические риски. При таком разделении под частными рисками 
обычно понимают любой риск формирующийся во внешней среде организации, а под стратегическими рисками - риск, образующийся в результате последствий воздействия частных рисков и оказывающий непосредственное воздействие на экономический результат хозяйственной деятельности - это риск снижения доходов и риск незапланированного повышения расходов [9]. В данной статье предлагается, методика сквозного управления рисками торговых предпринимательских структур, ориентированная на применение современных цифровых методов и инструментов. Основные этапы и элементы реализации предлагаемой методики представлены на рис. 1.

Реализацию данной методики предлагается проводить на основе базисных принципов сквозного управления рисками, детально рассмотренных в [10], основными из которых являются:

- Принцип обратной связи, в соответствии с которым должна осуществляться корректировка методов и инструментов управления рисками по результатам анализа и оценки эффективности применяемых методов и инструментов.

- Принцип учёта взаимного влияния рисков, в соответствии с которым управление ри- сками должно учитывать взаимное воздействие рисков и обеспечивать своевременную идентификацию предвестников и факторов риска.

- Принцип эффективности, в соответствии с которым все мероприятия по управлению рисками должны быть экономически обоснованы и не превышать по стоимости ожидаемый ущерб от активации рисков.

- Принцип полноты используемой информации в соответствии с которым в процессе риск-менеджмента должна использоваться и приниматься принимается во внимание вся доступная информация.

Принцип обратной связи реализуется корректировкой разрабатываемых мероприятий по результатам их воздействия на снижение ущерба от рисков и повышение эффективности предпринимательской деятельности. Принцип учёта взаимного влияния рисков учитывается в процессах формирования набора цифровых индикативных сервисов, идентификации и оценке рисков. Принцип эффективности учитывается при разработке мероприятий по управлению рисками и оценке целесообразности их реализации. Выполнение принципа полноты используемой информации обеспечивается применением современных облачных технологий и сервисов

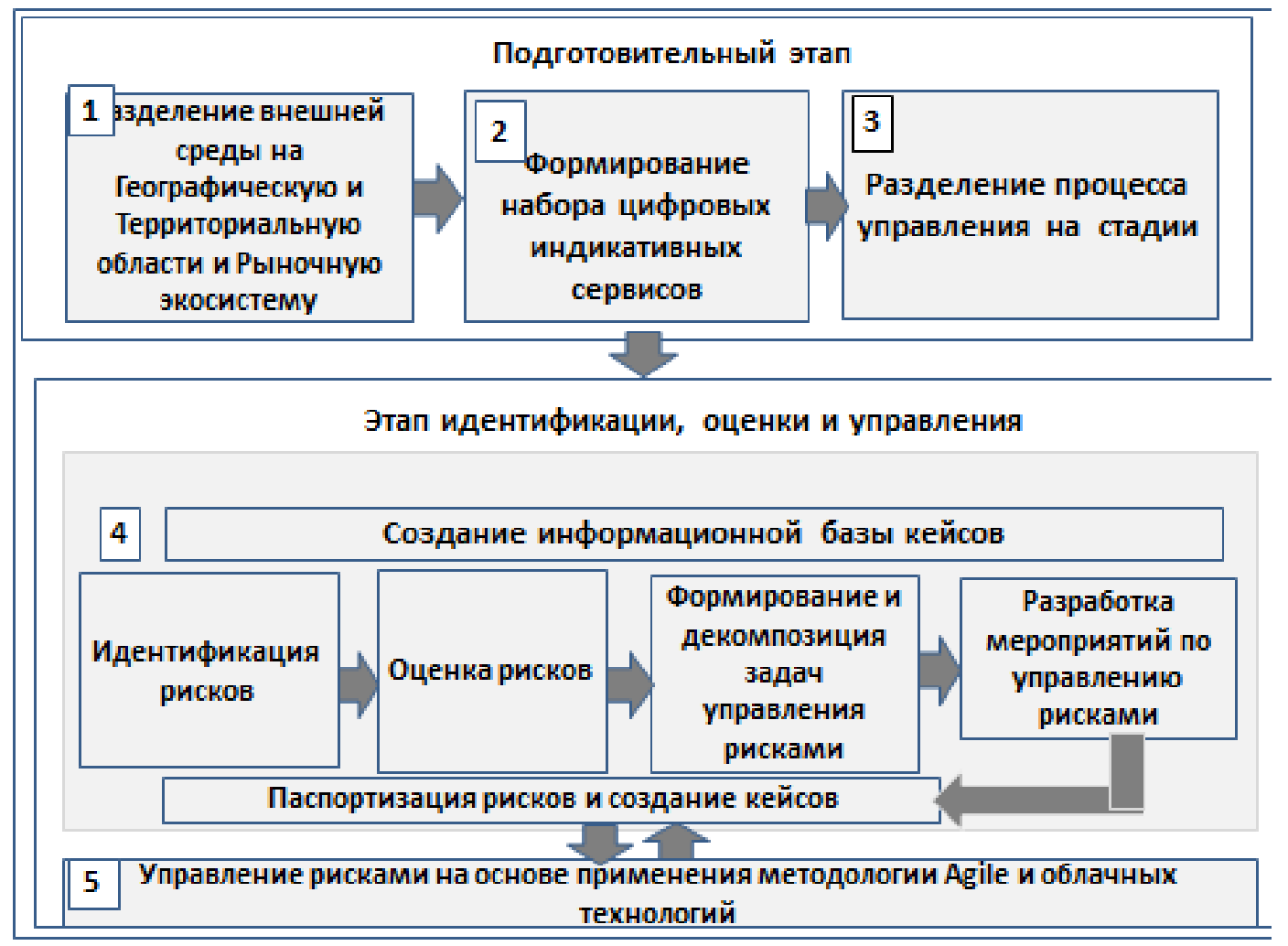

Puc. 1. Этапы и элементы методики сквозного управления частными рисками 
при использовании в процессе управления рисками методологии Agile.

Рассмотрим более подробно содержание каждого из модулей предлагаемой методики сквозного управления рисками.

Модуль 1 - Разделение внешней среды на Географическую и Территориальную области и Рыночную экосистему.

В данный модуле осуществляется структурирование внешней среды, состоящей из субъектов политической, экономической, социальной сфер деятельности общества и оказывающих воздействие на хозяйственную деятельность предпринимательских структур.

Географическая область объединяет субъекты внешней среды

по географическим признакам и включает в себя три уровня:

- Глобальный уровень, к которому относятся субъекты внешней среды оказывающие воздействие на компанию и находящиеся из вне юрисдикции РФ. К ним относятся международные организации, различные общественные, политические, экономические структуры иностранных государств и т.д.

- Страновой уровень, к которому относятся российские коммерческие и некоммерческие организации, органы федеральной власти и т.д.

- Региональный уровень, к которому относятся органы региональной власти и т.д.

Территориальная область объединяет субъектов внешней среды, относящихся к организациям, воздействующим на компанию по месту ее расположения и включает в себя:

- Контролирующие органы - такие как ФНС, ПФР, Роспотребнадзор и т.д

- Банк - осуществляющий РКО компании.

- Градообразующие предприятия (если таковые имеются на данной территории)

Рыночная экосистема включает в себя следующие субъекты:

Рыночная экосистема включает в себя следующие субъекты:

- Покупатель - лицо, приобретающее товары или услуги рассматриваемой организации

- Конкурент - организации ориентированная на тех же покупателей что и рассматриваемая организация.

- Партнер - лицо, имеющее партнерские отношения с рассматриваемой предпринимательской структурой по поставке товаров или услуг.
Модуль 2 - Формирование набора цифровых индикативных сервисов

Содержит перечень рекомендованных веб ресурсов способных предоставить дополнительную информацию о предпринимательских рисках формирующихся на различных уровнях внешней среды.

В данном модуле представлены наиболее популярные цифровые сервисы, применение которых позволяет решать задачи по идентификации рисков на основе обработки больших массивов данных. В качестве таких сервисов для идентификации рисков торгового предпринимательства предлагается использовать платформы Webhose, Skuuudle, WEBSERM, Buffer replay [11].

Платформа Webhose, в автономном режиме проводит информационный мониторинг веб-сайтов и соцсетей, сбор текстовых данных их структуризацию и анализ. Эта платформа может использоваться для прогнозирования валютных курсов, что особенно важно для торговых предпринимательских структур, занимающихся экспортно-импортными операциями. Использование этой платформы нацелено на снижение валютных рисков.

Интеллектуальную платформу Skuuudle peкомендуется применять для мониторинга ценовых изменений рекламных и пиар акций, а также для выявления новых товаров предлагаемых конкурентами. Использование этой платформы нацелено на совершенствование ценовой политики компании и снижение рисков падения выручки из-за ошибочной ценовой политики.

Важным элементом эффективного управления рисками торгового предпринимательства является анализ негативного контента в различных социальных сетях о компании, товаров и услугах. Для этих целей предлагается использовать платформу WEBSERM, работающую по технологии SERM (Search Engine Reputation Management), что в переводе с английского означает управление репутацией в поисковых системах. Этот сервис предлагает множество опций и программ, позволяющих настроить процесс сбора информации о негативном контенте и автоматизировать процесс выдвижения контраргументов он-лайн оппоненту в целях нейтрализации негативного отрицательного информационного фона и снижения репутационных рисков.

Другим важным элементом управления рисками торговых предпринимательских структур является использование новостных агрегаторов 
для управления предпринимательскими рисками, позволяющих аккумулировать информацию в соответствии с определенными ключевыми словами и настраиваемыми условиями. Для торговых предпринимательских структур для аккумулирования этой информации предлагается использовать платформу Buffer, применение которой нацелено на снижение операционных рисков товарно-договорной группы в частности применение этой платформы позволит снизить риски вызванные отсутствием в договорах учета возможных ценовых изменений, изменение рыночной конъектуры.

Модуль 3 - Разделение процесса управления на стадии

В данном модуле выделяются три стадии, характеризующие процесс зарождения и реализации и воздействия рисков, а именно:

- предрисковая стадия, предшествующую реализации риска;

- стадия реализации риска;

- стадия последействия, характеризуемая негативными последствиями реализации риска.

На предрисковой стадии реализуются превентивные мероприятия, нацеленные на снижение вероятности активации рисков и величину предполагаемого ущерба тот его воздействия. Данная стадия является наименее затратной при этом эффективность проводимых мероприятий обеспечивается степенью культуры риск-менеджмента в организации и в частности развитием в ней риск-ориентированной культуры, предусматривающей создание и организацию такой среды которая способствует выявлению, оценке и снижению рисков [12].

Стадия реализации риска наступает с момента активации риска и до окончания его воздействия. На этой стадии реализуются постактивационные мероприятия реактивного управления рисками. Целью данных мероприятий является снижение негативного воздействия риска за счет оперативной локализации риска и оперативной реализации мер противодействия риску. Важным фактором эффективности мероприятий на данной стадии управления рисками является оперативность и дисциплина выполнения мероприятий реактивного управления рисками на основе ранее разработанных регламентов внутреннего управления.

На стадии последействия осуществляется компенсация ущерба, полученного от воздействия реализовавшихся рисков, на основе при- менения и возможного уточнения заранее разработанных методов компенсации.

Предлагаемое разделение процесса воздействия рисков на три стадии, позволяет структурировать мероприятия риск-менеджмента с последующим созданием базы кейсов, каждый из которых включает в себя иерархическую систему задач, методов управления и мероприятий по управлению рисками.

\section{Модуль 4 - Создание информационной базы кейсов}

Данный модуль содержит базу кейсов управления рисками Географической и Территориальных областей. Каждый кейс содержит паспорт риска, задачи, методы управления данным риском и мероприятия которые целесообразно реализовать в процессе управления.

Заполнение паспорта риска и формулирование задач управления осуществляется на стадии идентификации и корректируется после оценки риска. После оценки, разрабатываются мероприятия по управлению данным риском, которые могут корректироваться по результатам оценки их эффективности.

Таким образом построение базы кейсов осуществляется в пять этапов

1. Этап идентификации рисков, на котором каждый идентифицированный риск получает идентификационный код, который указывается в паспорте риска.

2. Эman оценки рисков, на котором осуществляется оценка риска учитывающая его векторный характер, т.е. наличие двух параметров риска вероятности активации и размера возможного ущерба. Результаты оценки риска заносятся в его паспорт.

3. Этап определения интегральных задач и их декомпозиция на вектор задачи. На этом этапе выполняется процесс постановки интегральных задач (вектор-задач) и их декомпозиция на частные задачи (smart-задачи) определяющий направление действий конкретных подразделений в рамках решения интегральных задач.

Интегральными рисками хозяйственной деятельности любой предпринимательской структуры являются риск снижения выручки и риск роста издержек. Поэтому, для конкретных частных рисков в качестве интегральных задач является противодействие негативному воздействию одному из этих рисков.

4. Этап разработки мероприятий. На этом этапе определяются методы управления риском 
и мероприятия, обеспечивающие снижение параметров риска на каждой стадии его воздействия.

5. Этап паспортизации рисков и создание кейсов. На данном этапе окончательно формируется паспорт кейса с указанием идентифицированного риска, задач и методов управления им и создаются кейсы риска для каждого подразделения на каждой стадии управления риском. Все созданные кейсы заносятся в матрицу кейсов с уникальным идентификационным кодом, где число это номер риска, а буква обозначает подразделение компании которому принадлежит данный кейс.

Пример: Кейс 38Б, 38 - номер риска «Рост таможенных пошлин», «Б»- отдел бухгалтерии.

Модуль 5.- Управление рисками на основе методологии Agile и облачных технологий

В рамках предлагаемой концепции на предрисковой стадии и стадии активации риска предлагается применять гибкую методологию Agile, которая первоначально была предложена для разработки IT-проектов [13] и получила дальнейшее распространение на проекты в других сферах [14]. В данной работе предлагается использовать данную методологию для минимизации рисков путем сведения процесса управления рисками к серии коротких циклов называемых итерациями (спринтами).

Для управления рисками по методологии Agile формируются КУР (команда управления рисками) и АГ (аналитическая группа).

КУР включает в себя: специалисты отделов связанных с управлением рисками и скраммастера - специалиста Agile, в состав АГ предлагается ввести риск-менеджеров и бизнес аналитики.

Для реализации Agile на практике используют различные облачные сервисы, наиболее рас- пространенными из которых являются Jira, TFS, Version One, Rally Spreadsheet [15].

Для хранения и мониторинга состояния решения задач сквозного управления рисками предлагается использование облачного сервиса Яндекс Трекер. Применение этого сервиса обеспечивает структурирование задач, для каждой из которой заводится отдельная страница и легкий поиск информации на основе использования ключевых слов. Эффективность контроля обеспечивается наглядным представлением информации на дашборде. Благодаря указанным достоинствам данного сервиса обеспечивается экономия времени, а также снижение операционных ошибок участников процесса управления рисками за счет использования в сервере опций автоматических напоминаний [16].

Управление иерархической системой задач сквозного управления рисками на основе облачного сервиса Яндекс Трекер строится посредством осуществления декомпозиции вектор-задач на более мелкие и формирования бэклога. Критерий разбивки - выполнение данной задачи в течение одной итерации. Пример декомпозиции вектор-задачи «Разработать информационные базы альтернативных товаров» приведены таблице 1.

В этой таблице сложность выполняемых задач оценивается в условных единицах сложности - сторипоинтах (SP). Каждый член команды управления рисками (КУР) осуществляет оценку сложности каждой подзадачи по определенной шкале. Среднее арифметическое всех оценок принимается за итоговую оценку сложности в SP [17].

Сумма всех SP подзадач определяет трудоемкость решения данной Smart-задачи. Аналогичным образом определяется трудоемкость Vector-задач. Такой подход позволяет выявить и

Таблица 1. Пример декомпозиции вектор-задачи

\begin{tabular}{|c|c|c|c|}
\hline Vector-задача & Smart-задачи & Подзадачи & $\begin{array}{c}\text { Трудо } \\
\text { затраты }\end{array}$ \\
\hline \multirow{8}{*}{$\begin{array}{c}\text { Разработать информа- } \\
\text { ционные базы альтер- } \\
\text { нативных товаров } \\
\text { 30sp }\end{array}$} & \multirow{2}{*}{$\begin{array}{c}\text { Провести маркетинго- } \\
\text { вые исследования } \\
13 \mathrm{sp}\end{array}$} & Выполнить интернет исследование & $4 \mathrm{sp}$ \\
\hline & & Посетить выставки & 9 sp \\
\hline & \multirow{3}{*}{$\begin{array}{c}\text { Провести анализ мар- } \\
\text { кетинговых данных } \\
14 \mathrm{sp}\end{array}$} & Дать характеристику локации поставщиков & $3 \mathrm{sp}$ \\
\hline & & Определить ценовую категорию поставщиков & $5 \mathrm{cp}$ \\
\hline & & Дать заключение об условиях поставки & $6 s p$ \\
\hline & \multirow{3}{*}{$\begin{array}{c}\text { Командировать сотруд- } \\
\text { ника для переговоров } \\
7 \mathrm{sp}\end{array}$} & Определить дату командировки & $2 \mathrm{sp}$ \\
\hline & & Назначить командируемого сотрудника & $2 \mathrm{sp}$ \\
\hline & & Разработать план командировки & $3 s p$ \\
\hline
\end{tabular}


наглядно представить наиболее трудоемкие задачи процессов управления рисками и наиболее эффективно распределить ресурсы для решения этих задач [18].

Распространяя известные алгоритмы применения Agile на процессы риск-менеджмента можно построить модель сквозного управления рисками, представленную на рисунке 2 .

Для каждого кейса осуществляется матрёшечная декомпозиция вектор - задачи на смарт - задачи и т.д. Затем выделяются приоритетные задачи, которые должны быть решены в течение одного спринта. Планирование осуществляется следующим образом: в начале каждой итерации КУР оценивает трудоемкость декомпозированных задач из бэклога и выбирает необходимые задачи для данной итерации.

В начале каждого дня проводится встреча- стэндап где в течение 10-15 мин каждый член команды разъяряет план действий на день и ресурсы. По завершению итерации проводится демо-встреча КУР, на которой подводится итог с результатами достигнутых в данном спринте. По завершению демо-встречи проводится встречаретроспектива в рамках которой выявляются и формализуются положительные и отрицательные стороны завершенного в данном спринте процесса решения задач.

Контроль за выполнение этапов задач осуществляется за счет доски задач. В которой отслеживается состояние решения задач и статистика их выполнения накапливаемая с помощью трекера. На дашборде отражаются текущие результаты выполнения задач [18-19]. Пример дашборда иллюстрирующий процесс выполнения задач представлен на рисунке 3 .

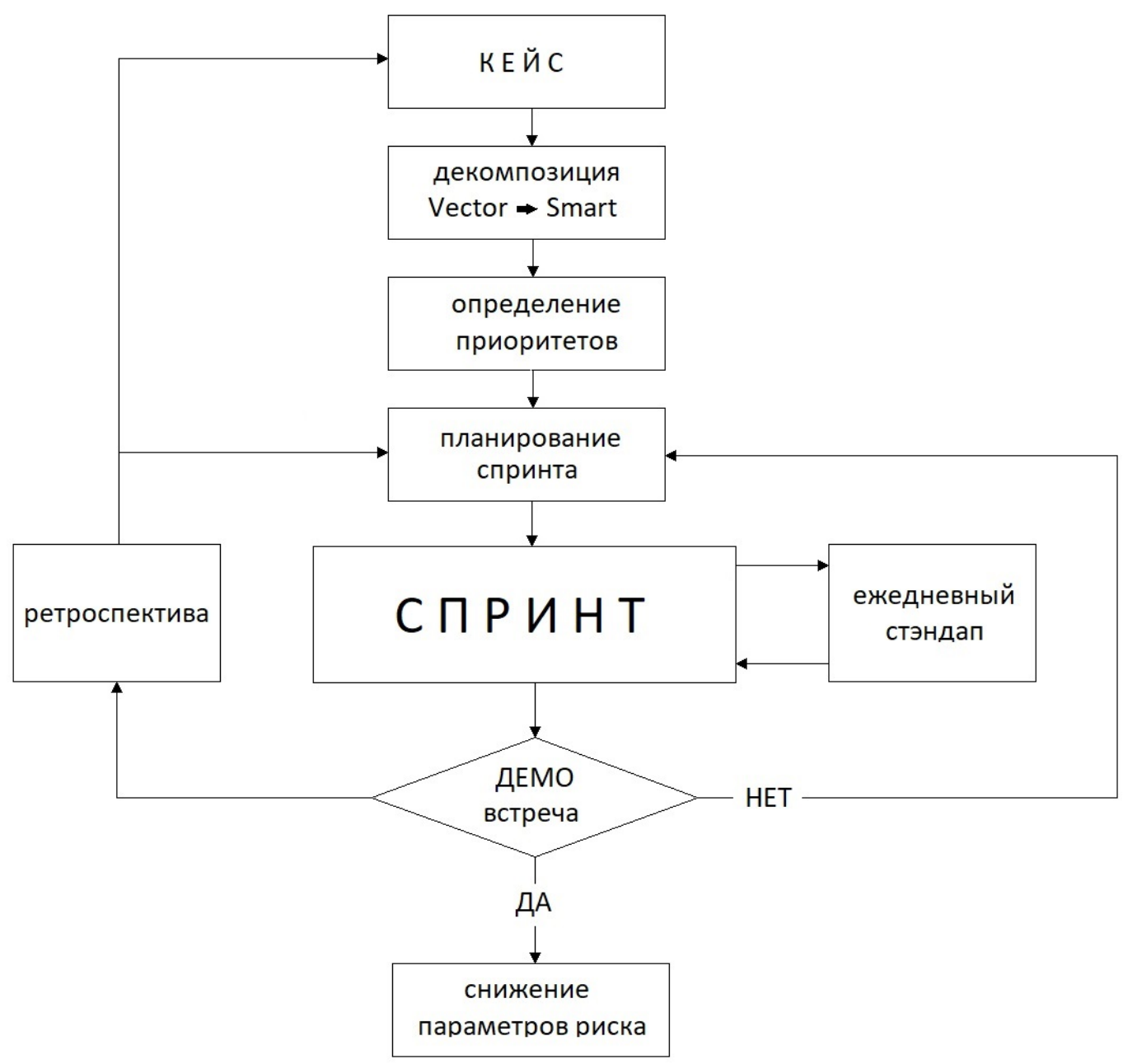

Puc. 2. Модель решения задач сквозного управления управления рисками на основе методологии Agile. 


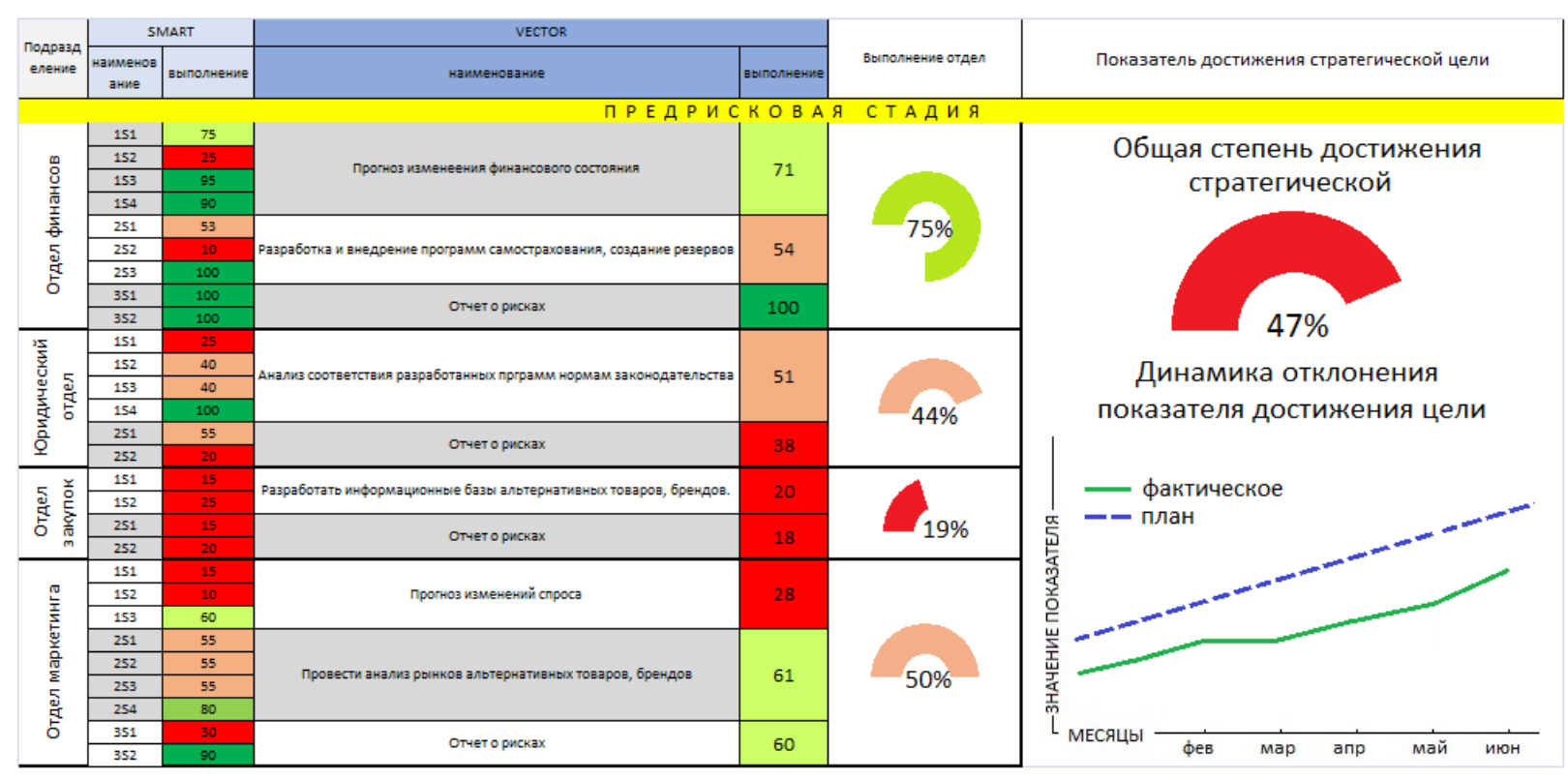

Puc. 3. Пример дашборда процесса сквозного управления рисками.

Таким образом, предлагаемая на основе использования современных облачных сервисов концепция сквозного управления частными рисками обеспечивает:

- автоматизацию и оперативность процессов управления.

- повышение объективности принятия решения.

- высокий уровень координации субъектов риск-менеджмента

- удобный и легкий доступ к информации о состоянии решения задачи по управлению рисками и параметрам идентифицированных и активированных рисков, каждый из которых имеет свой паспорт хранящийся в базе данных.

- Способствует развитию на предприятии риск-ориентированной культуры.
Следует отметить что реализация предлагаемой концепции сопряжена с дополнительными расходами на разработку освоение и внедрение современных цифровых технологий и сервисов, что требует от руководства предприятия четкого понимания необходимости инвестирования в совершенствования системы риск-менеджмента в целях повышения эффективности предпринимательской деятельности и конкурентоспособного развития данного предприятия. Однако в условиях характерной для настоящего времени экономической нестабильности и неопределённости и экспоненциально растущего объёма информации о потенциальных факторах риска такие расходы оправданы и компенсируются снижением потерь от негативного воздействия повышенных внешних рисков.

\section{Библиографический список}

1. Кунин В.А. Управление рисками промышленного предпринимательства (теория, методология, практика).СПб.: Изд-во Санкт-Петербургской академии управления и экономики, 2011.-184 с.

2. ГОСТ Р ИСО 31000-2010 [Электронный ресурс] // URL: http://gostrf.com/normadata/1/4293795/4293795643. pdf (дата обращения: 12.01.2021).

3. Костин К.Б., Кожухина К.А. Проблемы совершенствования управления рисками в предпринимательской деятельности//Научный журнал НИУ ИТМО. Серия Экономика и экологический менеджмент № 1, 2019. С. $171-180$.

4. Труды ИСА РАН: Алгоритмы. Решения. Математическое моделирование. Управление рисками и безопасностью / Под ред. С. В. Емельянова. - М.: Ленанд, 2014. - 102 с.

5. Вяцкова Н.С. Концептуальные и научные подходы управлению рисками предприятий // РИСК: Ресурсы, информация, снабжение, конкуренция.-2014.- № 2.- С. 260-264. 
6. Zemtsov S., Barinova V., Semenova R. (2019) The Risks of Digitalization and the Adaptation of Regional Labor Markets in Russia. Foresight and STI Governance, vol. 13, no 2, pp. 84-96.

7. Переславцева И.И. Управление рисками в условиях цифровой трансформации//Научный журнал Регион: системы, экономика управление. № 4 (47). 2019. С.207-209.

8. Орлова Л.Н., Ильин А. Б., Сизова Ю. С. Предпринимательские риски в условиях цифровизации экономики на примере России//Современная конкуренция. № 1(77). 2020. С. 76-85.

9. Михайловский Д.А. Методический подход к управлению стратегическими рисками в условиях высокой рыночной волатильности//Экономические науки. № 189. 2020. С. 55-63.

10. Кунин В.А, МихайловскийД.А. Концепция сквозного управления рисками торговых предпринимательских структур в условиях цифровизации российской экономики//Материалы III Международной научнопрактической конференции «Цифровая экономика и финансы» (ISPC-DEF 2020)//, [Электронный ресурс] URL: https://doi.org/10.2991/aebmr.k.200423.003, (Дата обращения:10.02.2021).

11. Rahbauer S. Adoption von Ökostrom durch kleine und mittelständische Unternehmen in Deutschland. [Электронный ресурс] URL: https://d-nb.info/1128309912/34 (дата обращения: 01.02.2021).

12. Яхваров Е.К. Формирование системы управления рисками в промышленных предпринимательских структурах: дисертация на соискание учёной степени канд. эк. наук: 08.00.05/ Е. К. Яхваров.- СПб., 2021. - 184 с.

13. Маслова В.М., Тубалова А. А. Необходимость Agile-мышления в системе современного менеджмента//Образование. Наука. Научные кадры. № 2. 2019. С. 160-163.

14. Лопатин Д.Н. Agile новый уровень мотивации в менеджменте//Динамика систем, механизмов и машин.№ 4. 2012. С31-34.

15. Разумное управление рисками в ходе цифровой трансформации//Исследование PWC из серии «Взгляд на риски». № 6. 2019.-42 с.

16. Яндекс Трекер. [Электронный ресурс] Инструменты для организации рабочего процесса. Режим доступа: URL: https://yandex.ru/tracker/ (Дата обращения 12.12.2020)

17. Акопян С. А. Управление проектами по принципам системы Agile, Scrum как один из методов управления проектами основанный на Agile//ECONOMICS. 2(33). 2017. C. 27-31.

18. Голубин А.Ю. Математические вопросы управления риском в базовых моделях страхования / Голубин Алексей Юрьевич.-М.: Анкил, 2020. - 538 с.

19. Антонов, Г.Д. Управление рисками организации: Уч. / Г.Д.Антонов, О.П.Иванова, В.М. Тумин.- М.: Инфра-М, 2018. -48 c.

20. Сердюченко О.П. Система управления рисками на предприятии: риск-менеджмент // Производственный менеджмент: теория, методология, практика. № 2. 2015. С. 172-176. 